\title{
Natural Course of Isolated Pulmonary Valve Stenosis in Pediatric Patients
}

\section{Güven Serçin $^{1 *}$, Öztarhan Kazım²}

${ }^{1}$ Department of Pediadric Nephrology, Marmara University,İstanbul , Turkey

${ }^{2}$ Istanbul Kanuni Sultan Süleyman Research and Teaching Hospital, Istanbul, Turkey

*Corresponding author: Güven Serçin, Department of Pediadric Nephrology, Marmara University,İstanbul, Turkey.

Received date: December 26, 2019; Accepted date: January 08, 2020; Published date: January 13, 2020

Citation: Serçin G., Kazım Ö. (2020) Natural Course of Isolated Pulmonary Valve Stenosis in Pediatric Patients. J Clinical Cardiology and

Cardiovascular Interventions, 3(2); DOI:10.31579/2641-0419/043

Copyright: () 2020 Güven Serçin, This is an open access article distributed under the Creative Commons Attribution License, which permits unrestricted use, distribution, and reproduction in any medium, provided the original work is properly cited.

\begin{abstract}
Aim:

The aim of our study is to be able to predict the prognosis of patients with isolated pulmonary valvular stenosis on the basis of age and degree of stenosis. Identification of the course of pulmonary stenosis of different age groups will significantly contribute both to the physicians and the relatives of the patient.

Material and Methods: 105 pediatric patients diagnosed with isolated pulmonary valvular stenosis were included in our study. We investigated the impact of the gradient of stenosis and the age at the time of diagnosis on the natural course of pulmonary stenosis. Mean follow-up time of the children was 19 months, $25.45 \pm 22.48$ months. The patients were divided into four groups over their trans-valvular gradient degrees and $<20 \mathrm{mmHg}$ was defined as transient, $20-39 \mathrm{mmHg}$ mild, $40-59 \mathrm{mmHg}$ moderate, $60 \mathrm{mmHg}$ and over as severe pulmonary stenosis.
\end{abstract}

Results: Between two to five months, none of the moderate stenosis cases progressed unlike other patient groups. The decline in the final gradient versus initial gradient was significant in children between two to five months and six months to two years in our study, and yet there was no significant change of initial and final gradients in patients under one month, and at two years and over.

Conclusion: It would be reasonable to conclude that the progression of pulmonary valvular stenosis is benign in patients with pulmonary valvular stenosis under $40 \mathrm{mmHg}$ of systolic gradient diagnosed after $6^{\text {th }}$ month of life.

Keywords: pulmonary valve, stenosis, gradient, natural course

\section{Introduction}

Valvular pulmonary stenosis (PS) is a condition characterized by obstruction to blood flow from the right ventricle to the pulmonary artery. PS can occur in isolation or be associated with other types of cardiac defects.

Valvular pulmonary stenosis was first described in 1761 by John Baptist Morgagni. It was reported that prevalence of pulmonary stenosis among all congenital cardiac malformations reached up to $10 \%$. [1,2].

Koretzky in 1969 first discussed the thickening of cusps from inside and a mucoid dysplastic valve. [3] Majority of patients with Ullrich -Noonan syndrome have this type of pulmonary stenosis. [4]

Patients with mild and moderate pulmonary stenosis with right ventricular pressure less than $60 \mathrm{mmHg}$ may survive asymptomatically. Patients who have symptoms like congestive cardiac failure or paroxysmal dyspnea episodes in the first years of their lives are in the highest risk groups. $[5,6]$
Right ventricular hypertrophy may develop in case right ventricular pressure during rest remains $>50 \mathrm{mmHg}$ for a long time. Valvular stenosis does not increase after the $2^{\text {nd }}$ decade, however, infundibular hypertrophy developing secondary to this condition may lead to the increase of the gradient. Progressive myocardial hypertrophy, fibrosis, dilatation and tricuspid regurgitation and dysrhythmia may develop. Mild or moderate pulmonary stenosis is hardly progressive. Life expectancy is normal when peak gradient is $<50 \mathrm{mmHg}$.

According to a study using cardiac catheterization, while systolic gradient from the right ventricle to the pulmonary arteries remained stable in majority of the patients, there was a significant increase in $14 \%$ and a significant decrease in $14 \%$ of the patients. A patient with mild pulmonary stenosis did not progress into severe pulmonary stenosis and $10 \%$ of the patients with moderate pulmonary stenosis reported to progress. It has been understood that most dramatic increase in stenosis occurred in children aged $<2$ years. Some of the patients had severe progression until the age 12 . It is very difficult to predict progression in children aged $<2$ years at diagnosis. [6]

There is limited number of studies conducted with the small age groups. Our hospital is a maternity hospital, all neonates are physically examined 
and echocardiography is applied for those with suspicion of any pathology. The aim of our study is to be able to predict the prognosis of patients with isolated pulmonary valvular stenosis on the basis of age and degree of stenosis.

\section{Methods}

105 patients under follow-up with the diagnosis of isolated pulmonary valvular stenosis at Bakırköy Maternity and Pediatric Hospital, Department of Pediatric Cardiology were included in this study.

Diagnosis of isolated pulmonary valvular stenosis was made by using General ElectricVivid S5brand Doppler Echocardiography device. Medical records of 105 patients, who were diagnosed and followed-up between 2000-2010 at the Cardiology Clinic, were retrospectively examined and findings on the first echocardiographic investigation performed on the patients, age of the patients at diagnosis, findings on the last echocardiographic investigation and the need for invasive intervention were noted.

Patients with incomplete investigation, additional cardiac abnormalities and with abnormal ventricular function were excluded from the study. Patients with small patent ductus arteriosus and patent foramen ovale were included in the study.

Severity of pulmonary stenosis is graded on the basis of the peak gradient between the right ventricle and the pulmonary artery. If the peak gradient measured during normal cardiac output is $<20 \mathrm{mmHg}$, the classification is defined as transient, between $20-39 \mathrm{mmHg}$ as mild, between $40-59$ $\mathrm{mmHg}$ as moderate and $\geq 60 \mathrm{mmHg}$ as severe. The classification based on the above-given gradients is valid only in patients with normal right ventricle systolic functions.
The study protocol was described to all patients and each participant provided informed consent and the study was approved by the local ethics committee of the university hospital (Protocol number: 09.2019.779).

\section{Statistical Analysis}

NCSS (Number Cruncher Statistical System) Statistical Software (Utah, USA) program was used for statistical analysis in assessing the data obtained from the study. While assessing the study data, besides descriptive statistical methods (Median, standard deviation, frequency), Kruskal-Wallis test was used for comparison of quantitative data and parameters among the groups. Mann-Whitney $U$ test was used for comparing the parameters in between two groups. Chi-square test was used for comparison of qualitative data. Intraclass correlation analysis was used to investigate the relations between the parameters. $p<0.05$ was taken for statistical significance.

\section{Results}

The study was performed on 105 pediatric patients, 52 male (49.5\%) and 53 female $(50.5 \%)$ with isolated pulmonary stenosis. Age at diagnosis of these children varied between one day to 13 years with an average age at diagnosis of $13.28 \pm 27.71$ months and median age of four months. The follow-up time of subjects ranged between seven days to ten years with an average of $25.45 \pm 22.48$ months and median of nine months.

Age at diagnosis was under one month in 31 patients (29.5\%), whereas it was between $2-5$ months in 27 patients $(25.7 \%)$, six months to two years in 33 patients $(31.4 \%)$ and $\geq 2$ years in 14 children (13.3\%) (Table 1$)$.

\begin{tabular}{|l|l|l|l|}
\hline \multicolumn{2}{|l|}{} & $\mathbf{n}$ & $\mathbf{\%}$ \\
\hline Age at diagnosis & $\leq \mathbf{1}$ month & 31 & 29,5 \\
\cline { 2 - 5 } & $\mathbf{2 - 5}$ months & 27 & 25,7 \\
\cline { 2 - 4 } & $\mathbf{6}$ months- 2 years & 33 & 31,4 \\
\cline { 2 - 4 } & $\geq 2$ years & 14 & 13,3 \\
\hline
\end{tabular}

Table 1: The distribution of children according to the age at diagnosis

Table 2 shows the changes in final gradients of 31 children with the age under one month at diagnosis. 19.4\% of these children (6/31) had transient initial gradient. In three of these children, gradient remained as transient at the final whereas the gradient was mild in three of them.

\begin{tabular}{|l|l|l|l|l|}
\hline \multicolumn{2}{|l|}{$\leq 1$ month (n=31) } & \multicolumn{3}{|l|}{ Follow-Up Time (month) } \\
\hline Initial gradient & Final Gradient & n & Min-Max & Mean \pm SD (Median) \\
\hline Transient $(\mathrm{n}=6)$ & Transient & 3 & $20-65$ & $45,33 \pm 23,03(51)$ \\
\cline { 2 - 5 } & Mild & 3 & $1-60$ & $25,66 \pm 30,66(16)$ \\
\cline { 2 - 5 } & Moderate & - & - & - \\
\cline { 2 - 5 } & Severe & - & - & - \\
\hline Mild $(\mathrm{n}=16)$ & Transient & 9 & $60-50$ & $16,22 \pm 13,69(12)$ \\
\hline
\end{tabular}




\begin{tabular}{|c|c|c|c|c|}
\hline & Mild & 5 & $18-62$ & $40,60 \pm 19,13(44)$ \\
\hline & Moderate & 1 & 49 & - \\
\hline & Severe & 1 & 2 & - \\
\hline \multirow[t]{4}{*}{ Moderate $(n=6)$} & Transient & - & - & - \\
\hline & Mild & 2 & $76-108$ & $92,0 \pm 22,63(92)$ \\
\hline & Moderate & 2 & $3-12$ & $7,5 \pm 6,36(7,5)$ \\
\hline & Severe & 2 & $3-7$ & $5,0 \pm 2,83(5)$ \\
\hline \multirow[t]{4}{*}{ Severe $(n=3)$} & Transient & - & - & - \\
\hline & Mild & - & - & - \\
\hline & Moderate & - & - & - \\
\hline & Severe & 3 & $0,23-0,66$ & $0,37 \pm 0,25(0,23)$ \\
\hline
\end{tabular}

Table 2: The distribution of initial and final gradients of children at the age of $\leq 1$ month at diagnosis

$51.6 \%$ of the children $(16 / 31)$ had mild initial gradients. Final gradient of nine children was transient whereas it remained mild in five patients, moderate in one and severe in one of them.19.4\% of the children $(6 / 31)$ had moderate initial gradient. Final gradient was mild in two of these children whereas it remained moderate in two of them and reached to severe level in two of them. $9.7 \%$ of the children $(3 / 31)$ had severe initial gradient. Gradients in all of these children remained severe.

The changes in final gradients of a total of 27 children at the age of two months to five months at diagnosis, $11.1 \%$ (3/27) of them had a transient initial gradient. The gradient remained transient in two of these children and in one child it remained mild.

$59.3 \%$ of the children $(16 / 27)$ had mild initial gradient. Ten of these children reached transient stage at the final, four patients remained mild and two children developed moderate PS.

$22.2 \%$ of them $(6 / 27)$ had moderate initial gradient. Final gradient was transient in one of these children, mild in three and moderate in two of them.

$7.4 \%$ of the children $(2 / 27)$ had severe initial gradient. Final gradient of both children was moderate. (Table 3 )

\begin{tabular}{|l|l|l|l|l|}
\hline \multicolumn{2}{|l|}{2} & \multicolumn{3}{l|}{ Follow-Up Time (month) } \\
\hline Initial gradient & Final Gradient & $\mathbf{n}$ & Min-Max & Mean \pm SD (Median) \\
\hline \multirow{4}{*}{ Transient (n=3) } & Transient & 2 & $8-20$ & $14,0 \pm 8,48(14)$ \\
\cline { 2 - 5 } & Mild & 1 & 6 & - \\
\cline { 2 - 5 } & Moderate & - & - & - \\
\cline { 2 - 5 } & Severe & - & - & - \\
\hline Mild (n=16) & Transient & 10 & $4-61$ & $21,10 \pm 22,86(10)$ \\
\cline { 2 - 5 } & Mild & 4 & $6-40$ & $15,75 \pm 16,26(8,5)$ \\
\cline { 2 - 5 } & Moderate & 2 & $6-39$ & $22,5 \pm 23,33(22,5)$ \\
\cline { 2 - 5 } & Severe & - & - & - \\
\hline Moderate (n=6) & Transient & 1 & 55 & $14,5 \pm 13,43(14,5)$ \\
\cline { 2 - 5 } & Mild & 3 & $3-28$ & - \\
\cline { 2 - 5 } & Moderate & 2 & $5-24$ & \\
\hline & & & & $19,0 \pm 13,89(26)$ \\
\hline
\end{tabular}




\begin{tabular}{|l|l|l|l|l|}
\hline & Severe & - & - & - \\
\hline Severe $(n=2)$ & Transient & - & - & - \\
\cline { 2 - 6 } & Mild & - & - & - \\
\cline { 2 - 5 } & Moderate & 2 & $4-29$ & $16,5 \pm 17,68(16,5)$ \\
\cline { 2 - 5 } & Severe & - & - & - \\
\hline
\end{tabular}

\section{Table 3: The distribution of initial and final gradients of children at the age of 2 months to 5 months at diagnosis}

According to the changes between the initial and final gradients of 33 children at the age of six months to two years at diagnosis;

Initial gradients were transient in $36.4 \%$ of them $(12 / 33)$ While final gradient of 10 in these children remained transient, two children's gradient ended at mild level.

Initial gradient in $33.3 \%$ of the children (11/33) was mild. While final gradient of three in these children was transient, final gradient of seven children remained mild and one of them reached to the moderate level.

Initial gradient in $21.2 \%$ of the children (7/33) was moderate. While final gradient of four children was mild, one of them remained moderate and two of them reached to severe level.

Inital gradient in $9.1 \%$ of the children (3/33) was severe. While final gradient of one child regressed to transient, two of them remained severe. (Table 4)

\begin{tabular}{|c|c|c|c|c|}
\hline \multicolumn{2}{|c|}{6 months -2 years $(n=33)$} & \multirow[b]{2}{*}{$\mathbf{n}$} & \multicolumn{2}{|c|}{ Follow-up Time (month) } \\
\hline Initial Gradient & Final Gradient & & Min-Max & Mean \pm SD (Median) \\
\hline \multirow[t]{4}{*}{ Transient $(n=12)$} & Transient & 10 & $7-41$ & $21,80 \pm 11,60(21)$ \\
\hline & Mild & 2 & $5-32$ & $18,5 \pm 19,09(18,5)$ \\
\hline & Moderate & - & - & - \\
\hline & Severe & - & - & - \\
\hline \multirow[t]{4}{*}{ Mild $(n=11)$} & Transient & 3 & $8-55$ & $28,67 \pm 24,01(23)$ \\
\hline & Mild & 7 & $6-120$ & $39,71 \pm 37,61(27)$ \\
\hline & Moderate & 1 & 16 & - \\
\hline & Severe & - & - & - \\
\hline \multirow[t]{4}{*}{ Moderate (n=7) } & Transient & - & - & - \\
\hline & Mild & 4 & $16-58$ & $33,5 \pm 17,92(30)$ \\
\hline & Moderate & 1 & 17 & - \\
\hline & Severe & 2 & 12 & 12 \\
\hline \multirow[t]{4}{*}{ Severe $(n=3)$} & Transient & 1 & 52 & - \\
\hline & Mild & - & - & - \\
\hline & Moderate & - & - & - \\
\hline & Severe & 2 & $3-7$ & $5,0 \pm 2,83(5)$ \\
\hline
\end{tabular}

Table 4: The distribution of initial and final gradients of children at the age of 6 months to 2 years at diagnosis

The change in the gradients of 14 children at the age of two years and over at diagnosis is shown in Table 5. 


\begin{tabular}{|c|c|c|c|c|}
\hline \multicolumn{2}{|l|}{$\geq 2$ years $(n=14)$} & \multirow[b]{2}{*}{$\mathbf{n}$} & \multicolumn{2}{|c|}{ Follow-up Time (month) } \\
\hline Initial Gradient & Final Gradient & & Min-Max & Mean \pm SD (Median) \\
\hline \multirow[t]{4}{*}{ Transient $(n=3)$} & Transient & 2 & $19-23$ & $21,0 \pm 2,83(21)$ \\
\hline & Mild & 1 & 29 & - \\
\hline & Moderate & - & - & - \\
\hline & Severe & - & - & - \\
\hline \multirow[t]{4}{*}{ Mild $(n=6)$} & Transient & 3 & $25-54$ & $41,0 \pm 14,73(44)$ \\
\hline & Mild & 3 & $16-58$ & $41,0 \pm 22,11(49)$ \\
\hline & Moderate & - & - & - \\
\hline & Severe & - & - & - \\
\hline \multirow[t]{4}{*}{ Moderate $(n=3)$} & Transient & - & - & - \\
\hline & Mild & 2 & $16-23$ & $19,5 \pm 4,95(19,5)$ \\
\hline & Moderate & - & - & - \\
\hline & Severe & 1 & 18 & - \\
\hline \multirow[t]{4}{*}{ Severe $(n=2)$} & Transient & - & - & - \\
\hline & Mild & - & - & - \\
\hline & Moderate & - & - & - \\
\hline & Severe & 2 & $1-45$ & $23,0 \pm 31,11(23)$ \\
\hline
\end{tabular}

Table 5: The distribution of initial and final gradients of children at the age of $\geq 2$ years at diagnosis

Initial gradient in $21.4 \%$ of them (3/14) was transient. Two of them remained transient at the end and one child had mild final gradient. Initial gradient in $42.9 \%$ (6/14) was mild. Three of these children had transient final gradient and three of them had mild final gradient.

Initial gradient in $21.4 \%$ (3/14) was moderate. While the gradient of two of these children was mild at the final stage, one of them reached to the severe level.

Initial gradient in $14.3 \%$ of the children $(2 / 14)$ was severe. Final gradient of both children remained severe.

There was no statistically significant difference at the final gradient versus the initial gradient in neonates $(\mathrm{p}>0.05)$.
The decrease observed at the final gradient versus the initial was highly statistically significant in children at the age of 2-5 months at diagnosis $(\mathrm{p}<0.01)$.

The decrease observed at the final gradient versus the initial was statistically significant in children at the age of 6 months- 2 years at diagnosis $(\mathrm{p}<0.05)$.

There was no statistically significant difference at the final gradient versus the initial gradient in children at the age of two years and over at diagnosis $(\mathrm{p}>0.05)$ (Table 6$)$ 


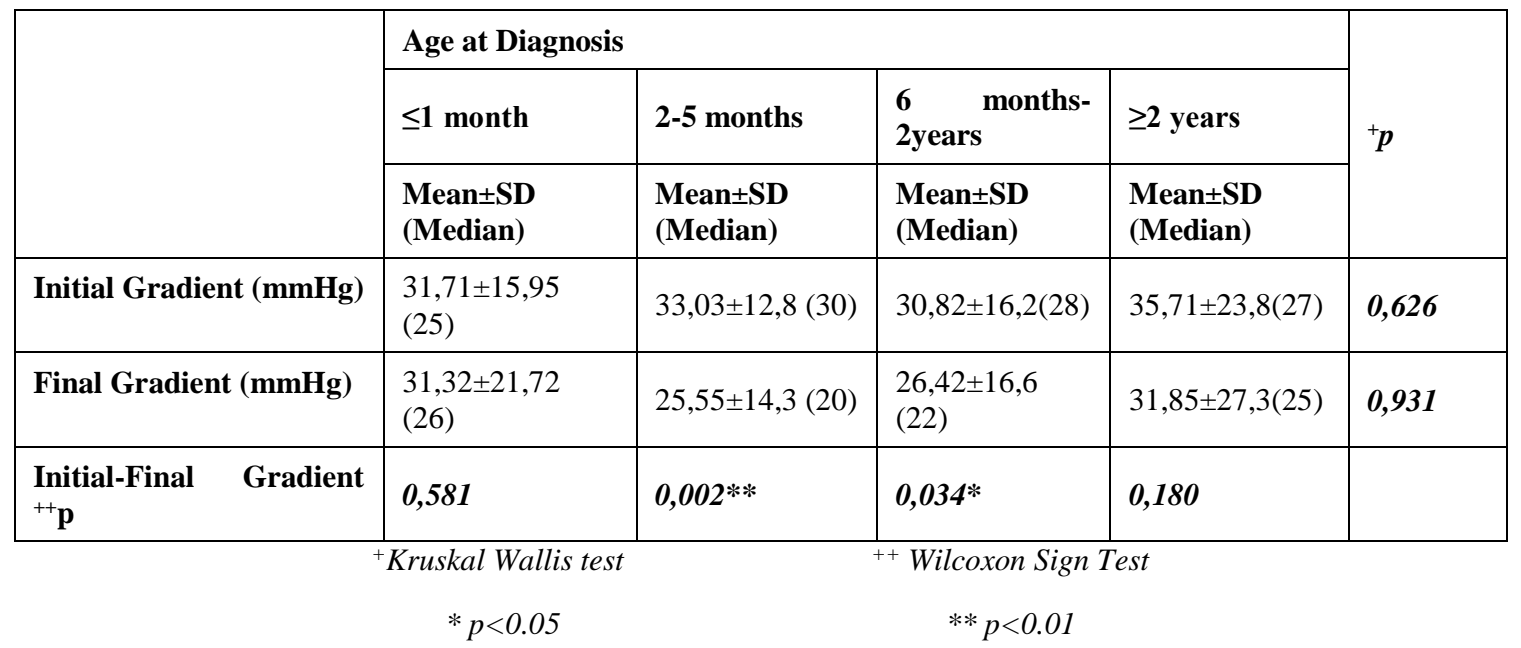

Table 6: Overview of initial and final gradients of children according to their ages at diagnosis

Balloon valvuloplasty was performed on 11 patients in our study. Six of these patients (54\%) had moderate pulmonary stenosis according to initial gradient whereas five of them $(46 \%)$ presented with severe stenosis. Age of onset was under one month in three of the patients with severe pulmonary stenosis whereas one was at the age of ten months and the other at four years. Three patients with moderate initial gradients were at the age of one month and under, whereas the other three were between six months and to two years. Age of onset of patients having undergone balloon valvuloplasty was 9,8 \pm 15 months with a follow-up time of 5,8 $\pm 5,9$ months.

\section{Discussion}

Studies investigating the progression of stenosis severity in patients with pulmonary valvular stenosis presenting with intact septum do not report any need for cardiac surgery in $95 \%$ of the patients with a mild initial gradient in 25-year follow-up. The gradient of moderate or severe pulmonary stenosis tends to increase with age.

Mild to moderate pulmonary valvular stenosis cases are generally associated with a good prognosis. Severe and moderate stenoses with an onset before four years of life are reported to associate with secondary infundibular stenosis resulting in a progressive worsening of the condition and poor prognosis. Severe obstruction may impair the right ventricle in years causing arrhythmias and premature mortality.

In a study on 51 infants with asymptomatic pulmonary valvular stenosis, Anandet al. observed a rapid progression in six (15\%) of the patients. Three of these six patients reached surgical gradient level in six months and the remaining three after the age of two [7].

In another study on 174 patients, Gielenet al reported 3\% progression to severe stenosis in patients with a transient initial stenosis. The ratio of patients with mild and moderate stenosis was $10 \%$ and $9 \%$, respectively [8].

Another study reports cardiac catheterization in 184 of 261 patients with a significant gradient increase in only $26(14 \%)$ of the patients. Only 22 children under the age of two years were included in this study. Investigators concluded that children under four years have a higher risk of progression when their initial gradient is already high
[9]. The second 'Natural History Study of Congenital Heart Defects' trial followed patients with a gradient of $25 \mathrm{mmHg}$ and under not detecting any increase in any of the subjects [10]. Both studies and other similar trials concluded that mild pulmonary stenosis is a static lesion that rarely progresses.

In a study by Rowland et al., 147 patients were followed-up detecting an increase equal to $20 \mathrm{mmHg}$ and more in gradients of 16 patients (29\%) out of 56 under one month of age. $80 \%$ of 21 patients under one month with a initial gradient under $20 \mathrm{mmHg}$ remained stable, $63 \%$ of 19 patients with a mild initial gradient finished the study with a mild gradient level. Rest of the patients progressed to moderate and/or severe stage. [11].

Mild and moderate pulmonary valvular stenosis cases in our study between two months to two years have a benign prognosis. None of the patients with a transient initial gradient developed severe stenosis. $6 \%$ of the patients with mild stenosis and $23 \%$ of the ones with moderate stenosis developed severe stenosis. There is no difference in terms of time of follow-up of these patients. 11 of 105 patients required balloon valvuloplasty, all of whom were diagnosed under four years of life. In six of the 31 patients $(29,5 \%)$ under one month, initial gradient was $<20 \mathrm{mmHg}$, all of whom reached mild and transient stage by the end of the study. Gradient level of $87 \%$ of 16 patients with a mild initial gradient remained the same or regressed. Rest of the patients progressed to moderate and severe stage. Corroborating with Rowland's study, the subjects in our study under one month with severe initial gradients required surgery.

Derrick et al reported that $25 \%$ of the patients diagnosed with mild pulmonary stenosis at the neonatal period developed significant stenosis in the time to follow to the degree that required surgery in half of them [12] in our study, two of the 16 patients (13\%) diagnosed with mild pulmonary stenosis during neonatal period presented with a progressed stenosis. Nevertheless, none of these patients required balloon valvuloplasty.

While $88 \%$ of patients with mild stenosis between two to five months have not presented with a progression in the level of their stenosis, $12 \%$ progressed to moderate stenosis. $34 \%$ of moderate stenosis remained stable whereas $66 \%$ presented a decrease in the degree of 
stenosis. All severe cases regressed to moderate stage. None of the patients in this age group progressed. $9 \%$ of the patients in the age group of six months to two years with mild stenosis progressed to moderate stage whereas $91 \%$ remained stable or regressed to transient stenosis. While $28 \%$ of the moderate group progressed, $14 \%$ remained stable and $57 \%$ regressed to a lesser degree of stenosis. Mild stenosis cases above two years did not progress and only $33 \%$ of moderate cases progressed to a higher degree of stenosis. As far as the subjects between two to five months are concerned, none of the moderate stenosis cases progressed unlike other patient groups. The decline in the final gradient versus initial was significant in children between two to five months and six months to two years in our study, and yet there was no significant change of initial and final gradients in patients under one month, and at two years and over. This may be explained with the fact that the body weight increases by two folds between two to five months, during which cardiovascular structures grow with the body, whereas the timeline between six months and two years is the period where the body grows exponentially. Valve outgrowth unproportional to somatic growth may be responsible for gradient changes requiring close follow-up.

\section{Conclusion:}

It would be reasonable to conclude from the study that the progression of pulmonary valvular stenosis is benign in patients with pulmonary valvular stenosis under $40 \mathrm{mmHg}$ of systolic gradient diagnosed after 6th month of life. Patients diagnosed at neonatal period should be followed more closely and frequently regardless of the degree of their stenosis.

\section{References}

1. Abbott ME. (1936) Atlas of congenital cardiac disease. New York: American Heart Association,
2. Morgagni JB. De Sedibus et Causis Morborum (The seats and causes of diseases). Venice: Remondini vol 1, 1761:154

3. Koretzky E D, Moller J H, Korns M E, Schwartz C J, Edwards J E. (1969) Congenital pulmonary stenosis resulting from dysplasia of valve. Circulation 40: 43-53

4. Rodriguez- Fernandez H L, Char F, Kelly D T, Rowe R D. (1972) The dysplastic pulmonic valve and the Noonan syndrome. Circulation 46(suppl II): 98

5. Rowe R D. (1978) Pulmonary stenosis with normal aortic root. In : Keith J D, Rowe R D, Vlad P, eds. Heart disease in infancy and childhood,3 rd edn. New York: Macmillan, b; 761-788

6. Nugent E W, Freedom R M, Nora J J,Ellison R C, Rowe R D, Nadas A S. (1977) Clinical course in pulmonary stenosis. Circulation 56 (suppl I): 38-47

7. Anand R, Mehta A V. (1997) Natural history of asymptomatic valvar pulmonary stenosis diagnosed in infancy. Clin Cardiol 20(4): 377-380

8. Gielen H, Daniéls O, van Lier H. (1999) Natural history of congenital pulmonary valvar stenosis:an echo and doppler cardiographic study. Cardiol Young 9(2): 129-135

9. Nugent E W, Freedom R M, Nora J J,Ellison R C, Rowe R D, Nadas A S. (1977) Clinical course in pulmonary stenosis. Circulation 56 (suppl I): 38-47

10. Hayes C J, Gersony W M, Driscoll D J,et al. (1993) Second Natural History Study of Congenital Heart Defects: results of treatment of patients with pulmonary valvar stenosis. Circulation 87 (suppl I): 28-37

11. Rowland Daniel G, Hammill William W, Allen Hugh D, Gutgesell Howard P. (1997) Natural course of isolated pulmonary valve stenosis in infants and children utilizing doppler echocardiography. Am J Cardiol 79: 344-349

12. Derrick G, Banhoeffer P, Anderson RH. (2010) Pulmonary stenosis In: Anderson RH, Baker EJ, Penny DJ, Redington AN, Rigby ML, Wernovsky eds. Paediatric Cardiology, 3rd edn. Philedelphia: Churchill Livingstone, p 895-917

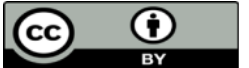

This work is licensed under Creative Commons Attribution 4.0 License

To Submit Your Article Click Here: Submit Article

DOI:10.31579/2641-0419/043
Ready to submit your research? Choose Auctores and benefit from:

* fast, convenient online submission

* rigorous peer review by experienced research in your field

* rapid publication on acceptance

* authors retain copyrights

* unique DOI for all articles

* immediate, unrestricted online access

At Auctores, research is always in progress.

Learn more www.auctoresonline.org/journals/clinical-cardiology-andcardiovascular-interventions 